\title{
Some Biochemical Effects of Chloral Hydrate in an Infant with a Tyrosinemia-like Syndrome
}

\author{
R. W. E. WATTS, ${ }^{(17)}$ R. A. CHALMERS, M. M. LIBERMAN, AND A. M. LAWSON
}

Division of Inherited Metabolic Diseases, and Division of Clinical Chemistry, Medical Research Council Clinical Research Centre, and Department of Pediatrics, Northwick Park Hospital, Watford Road, Harrow, Middlesex, England

\section{Extract}

An infant with a combination of clinical and biochemical features resembling those found in hereditary tyrosinemia ("inborn hepatorenal dysfunction with tyrosyluria") but with spontaneous recovery is described briefly. The child also had severe congenital hydrocephalus, and was being given chloral hydrate. She was not treated by restriction of dietary phenylalanine and tyrosine. The abnormal aromatic aciduria was unaltered by ascorbic acid administration. The results of loading tests with phenylalanine, tyrosine, and sodium 4-hydroxyphenylpyruvate are compatible with the existence of a partial block in the oxidation of 4-hydroxyphenylpyruvate to homogentisate which was exacerbated by administering chloral hydrate.

\section{Speculation}

It is suggested that an abnormality of the patient's 4-hydroxyphenylpyruvate dioxygenase (EC. 1.13.11.27) system rendered it susceptible to inhibition by chloral hydrate or its metabolites in vivo. Such an abnormality could be due to a genetically determined abnormality of the enzyme protein or exposure to an unidentified infective or toxic agent in utero.

Tyrosyluria, (the excretion of 4-hydroxyphenylpyruvate, 4-hydroxyphenyllactate and 4-hydroxyphenylacetate with hypertyrosinuria), occurs in: (l) some normal infants, especially premature babies, (2) scurvy, (3) tyrosinosis (12), (4) hepatic cytosol tyrosine: 2-oxoglutarate aminotransferase (EC 2.6.1.5) deficiency (13), (5) hereditary tyrosinemia ("inborn hepatorenal dysfunction with tyrosyluria") (14).

This paper reports a patient in whom indirect biochemical evidence of reduced 4-hydroxyphenylpyruvate dioxygenase (EC. 1.13.11.27) activity was associated with chloral hydrate administration. During the early months of life, the child's plasma and urinary amino acid patterns, mellituria, and phosphaturia resembled those seen in hereditary tyrosinemia. Some of these data were reported in preliminary form before one major urinary metabolite had been identified as trichloroethanol glucuronide (2).

\section{CASE REPORT AND BIOCHEMICAL INVESTIGATIONS}

\section{CLINICAL HISTORY}

The patient was the first child of healthy 30 -year-old nonconsanguineous Caucasian parents and the mother had received only routine drug medications (iron and folate preparations) during her pregnancy. There was no family history of congenital neurologic abnormalities. The pregnancy proceeded normally until the 32 nd week of gestation, as judged by the date of the last menstrual period, when the membranes ruptured spontaneously, and she was delivered of a live girl after a total labor of $12 \mathrm{hr}$.

The infant's Apgar score was 4 at 1 min, 8 at 10 min. Intubation and intermittent positive pressure respiration were needed because of apnea. She weighed $1.99 \mathrm{~kg}$ (50th percentile) at 32 weeks (4), and the head circumference was $36 \mathrm{~cm}$ (more than the 97 th percentile value). The head appeared to be hydrocephalic with the cranial sutures widely separated and a large skull defect $(10 \times 8$ $\mathrm{cm}$ ) in the region of the posterior fontanelie. There were no other physical abnormalities except for a small cavernous hemangioma on the left forearm. An episode of mild transient respiratory distress which lasted for $3 \mathrm{hr}$ occurred immediately after birth, and was probably related to hypothermia after the initial resuscitation. The child was icteric for the first 9 days of life, hyperbilirubinemia reaching a maximum value $(271 \mathrm{mmol} / \mathrm{liter})$ on the fourth day of life. This was treated with phototherapy. The infant's karyotype was that of a normal female (XX). The appearance of the simple $\mathrm{X}$-rays of the skull were those of congenital hydrocephalus. $x$-Ray films of the spine, chest, and intravenous urogram were normal. The following were normal: fundoscopy and transillumination of the head, full blood count, blood immunoglobulin M levels, and rubella, cytomegalovirus, and toxoplasma titers. Initially, the ventricular cerebrospinal fluid contained a normal amount of protein $(0.6 \mathrm{~g} /$ liter $)$. The infant developed a Pseudomonas conjunctivitis, and air encephalography was therefore postponed until this infection had completely resolved. The appearances of the encephalograms when she was 2.5 weeks old suggested agenesis of the corpus callosum and absence of the septum pellucidum. An electroencephalogram showed very low voltage with very little development of rhythmic activity. These observations indicated only a limited amount of cortical activity, and it was considered that the insertion of a Spitz-Holter valve would serve no useful purpose.

The child was observed to be abnormally pale when she was 10 weeks old, and further investigation was therefore instituted. The results of this were as follows: hemoglobin, $8.1 \mathrm{~g} / 100 \mathrm{ml}$; packed cell volume, $23.6 \%$; total white cell count, 13,400 cells $/ \mathrm{mm}^{3}$; reticulocytes, 8\%; hemoglobin electrophoresis normal; haptoglobulins absent; no clinical or laboratory evidence of abnormal intravascular coagulation. Blood urea, serum sodium, potassium, bicarbonate, bilirubin, total serum protein, albumin, osmolarity, and aspartate transaminase were all normal. The child was normocalcemic and normophosphatemic. There was no increased excretion of urobilinogen. Glucose, fructose, and galactose were detected in the urine on several occasions. There was also a generalized amino aciduria with a disproportionately high excretion of methionine. These observations were first made by qualitative thin layer chromatography supported by microbiologic assays when the child was about 16 weeks old. The radiologic appearances of mild rickets were apparent when the child was 28 weeks old in spite of an adequate intake of vitamin D (600-800 $\mathrm{U} / 24 \mathrm{hr}$ ). The urinary excretions of vanilmandelic acids and $\delta$-aminolevulinic acid were not increased.

The child's clinical condition remained essentially unaltered but her serum transaminase level increased to $751 \mathrm{U} /$ liter when she was 20 weeks old. The histologic appearances of a percutaneous liver biopsy (at 21 weeks of age) were normal, tests for Australia antigen were negative, and bromosulfophthalein retention $=16 \%$ at $45 \mathrm{~min}$.

The plasma alkaline phosphatase was 31.6 King-Armstrong (KA) $\mathrm{U}$ and $44.4 \mathrm{KA} \mathrm{U}$ when she was about 16 weeks and 20 weeks old, respectively. The serum transaminase level had returned to normal when she was 24 weeks old.

The results of an oral fructose $(1 \mathrm{~g} / \mathrm{kg}$ body weight) tolerance test at the age of 210 days were normal. 


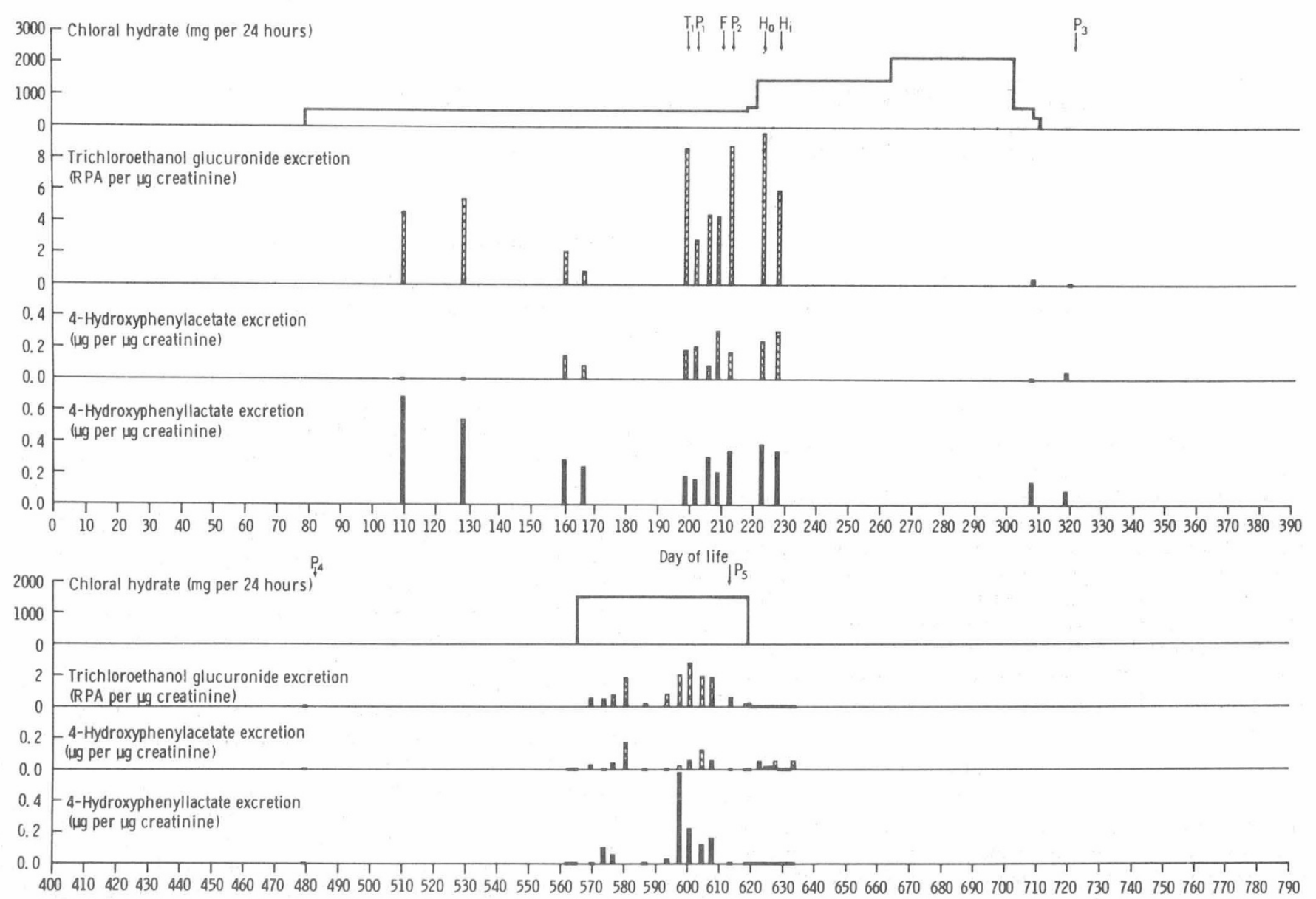

Day of life

Fig. 1. The excretion of 4-hydroxyphenyllactate, 4-hydroxyphenylacetate, and trichloroethanol glucuronide, and the periods and dosage of chloral hydrate. The vertical arrows mark the days on which the load tests were performed. $T_{1}$ : tyrosine load; $P_{1}, P_{2}, P_{3}, P_{4}, P_{5}$ : first to fifth phenylalanine loads; $F$ : fructose tolerance. $H_{o}$, oral 4-hydroxyphenylpyruvate load test; $H_{i}$, intravenous 4-hydroxyphenylpyruvate load test. Units of the trichlorethanol glucuronide excretion are given as RPA per $\mu$ g creatinine, where RPA is the peak area relative to $n$-tetracosane internal standard. When the factor relative to $N$-tetracosane is unity, the RPA is equal to $\mu \mathrm{g}$.

Chloral hydrate was given as a sedative from the 79th to the 309th days of life. The drug was also given when the child was between 565 and 619 days old (Fig. 1).

Amino acid analysis of urine, plasma, and cerebrospinal fluid using anion-exchange chromatography (Dr. V. G. Oberholzer) showed a generalized amino aciduria in the presence of hyperaminoacidemia, with the high plasma level of methionine being most striking. The amino acid concentration in the cerebrospinal fluid reflected those in the plasma. These abnormal levels had largely reverted to normal by the time the patient was 28 weeks old.

Acidic metabolites (with $\mathrm{pK}_{a}$ values of less than about 5.5) in urine were determined using quantitative extraction and gas chromatographic methods $(3,8)$, with mass spectrometric identification of the eluted metabolites (11). Results showed an elevated excretion of 4-hydroxyphenyllactic acid which increased markedly, with concomitant 4-hydroxyphenylpyruvic and 4-hydroxyphenylacetic acidurias, when oral loading tests with L-phenylalanine (100 $\mathrm{mg} / \mathrm{kg}$ body weight) and L-tyrosine (100 $\mathrm{mg} / \mathrm{kg}$ body weight) were performed. There were no other abnormalities in organic acid excretion, including phenylalanine metabolites, either before or during these loading tests, except for an elevated lactic acid and phosphate and the excretion of trichloroethanolglucuronide and trichloroacetic acid, the latter both being metabolites of chloral hydrate. The 4-hydroxyphenyllactic aciduria was not responsive to L-ascorbate administration (100 mg daily by mouth). The excretion of this acid, and of 4-hydroxyphenylpyruvic and 4-hydroxy- phenylacetic acids, also increased during oral $(100 \mathrm{mg} / \mathrm{kg}$ body weight) and intravenous ( $20 \mathrm{mg} / \mathrm{kg}$ body weight) 4-hydroxyphenylpyruvate loading tests. However, the total amounts of each metabolite excreted were similar to those observed in a similarly loaded adult control subject.

Figure 1 shows the periods of abnormal organic aciduria, days of life on which loading tests were performed, and periods of chloral hydrate administration. Abnormal responses to oral loading with $\mathrm{L}$-phenylalanine were observed during tests $P_{1}, P_{2}, P_{3}$, and $P_{5}$ and also during the tyrosine load test $\left(\mathrm{T}_{1}\right)$, the metabolism of both phenylalanine and tyrosine being grossly impaired (Figs. 2 and 3; Table 1). A normal response to oral loading with L-phenylalanine was noted during test $P_{4}$, when chloral hydrate was not administered, and it was apparent that the abnormal organic aciduria and loading test responses were associated with the periods of drug administration.

The excretion of 4-hydroxyphenyllactic and 4-hydroxyphenylpyruvic acids and of the phenylalanine metabolites, phenyllactic, phenylpyruvic, and 2-hydroxyphenylacetic acid, are not observed during loading tests with phenylalanine or tyrosine in normal subjects. Additionally, 6 nonhydrocephalic mentally retarded children and one mentally normal child who were taking chloral hydrate and 10 hydrocephalic children who were not receiving the drug excreted no detectable organic acids associated with impaired tyrosine and phenylalanine metabolism. All of the subjects receiving chloral hydrate excreted the anticipated large amounts of 


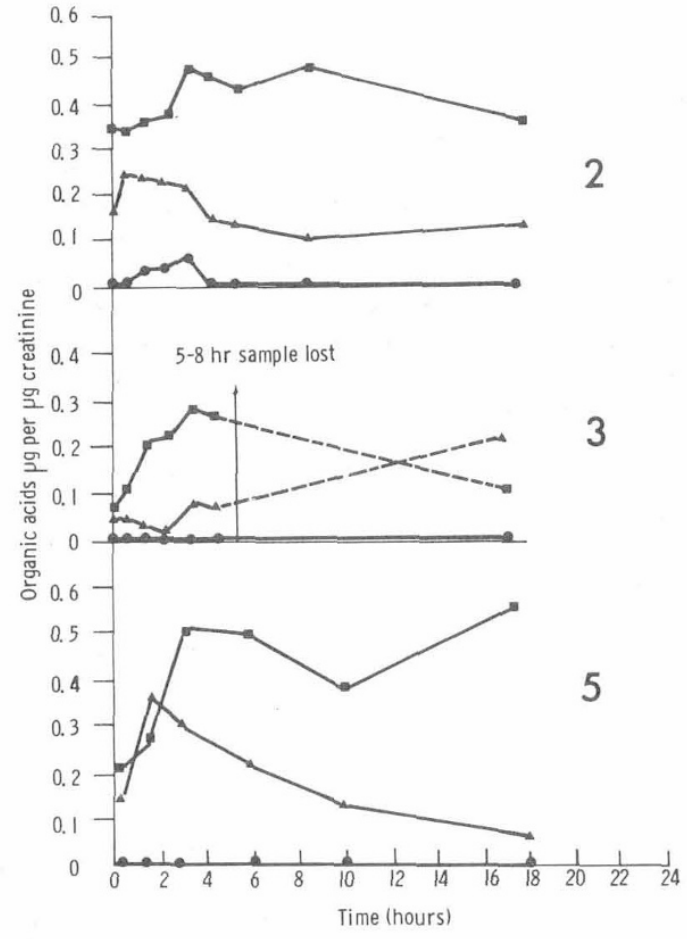

Fig. 2. The excretion of 4-hydroxyphenyllactate (- yphenylacetate ( $\mathbf{\Delta})$, and 4-hydroxyphenylpyruvate (-) during the $24 \mathrm{hr}$ after one oral dose of L-phenylalanine ( $100 \mathrm{mg} / \mathrm{kg}$ body weight) in the second (2), third (3), and fifth (5) phenylalanine load tests. These tests were performed on the 213,321 , and 614 th days of life, respectively. The urine collections were made by voluntary voiding and the experimentally determined points are shown at the middle of each urine collection period. trichloroethanolglucuronide but none excreted amounts of trichloroacetic acid that could be detected by specific and sensitive selected ion monitoring in the mass spectrometer.

\section{DISCUSSION}

The child appeared to thrive normally except for the increasing hydrocephalus, and although the serum transaminase level rose transiently when she was about 20 weeks old, there was no histologic evidence of liver disease.

The association of generalized hyperamino acidemia and hyperamino aciduria with marked hypermethioninemia, mellituria, and the abnormal excretion of aromatic acids. which had persisted in spite of ascorbic acid medication, suggested a diagnosis of hereditary tyrosinemia. A further liver biopsy to obtain tissue for enzyme assays could not be justified on ethical grounds.

The abnormal aromatic aciduria decreased and finally disappeared after chloral hydrate administration was stopped (Fig. 1). The renal tubule reabsorption defect had also disappeared by this time. The possible association of these changes with chloral hydrate was further investigated during a second period when the drug was given. The aromatic aciduria reappeared without the renal tubular defects. Similarly, the results of the phenylalanine load tests which were initially abnormal but which had reverted to a normal pattern when the chloral hydrate administration was stopped, again became abnormal. Phenylalanine and tyrosine load tests are not associated with aromatic aciduria in normal subjects, and the results of these tests in the present patient (Figs. 2 and 3 ) are compatible with reduced 4-hydroxyphenylpyruvate dioxygenase activity.

The sustained rise in plasma phenylalanine during the first phenylalanine load test, and the increase in plasma tyrosine during the tyrosine load test (Table 1) are compatible with some degree of impaired conversion of phenylalanine to tyrosine, and of tyrosine

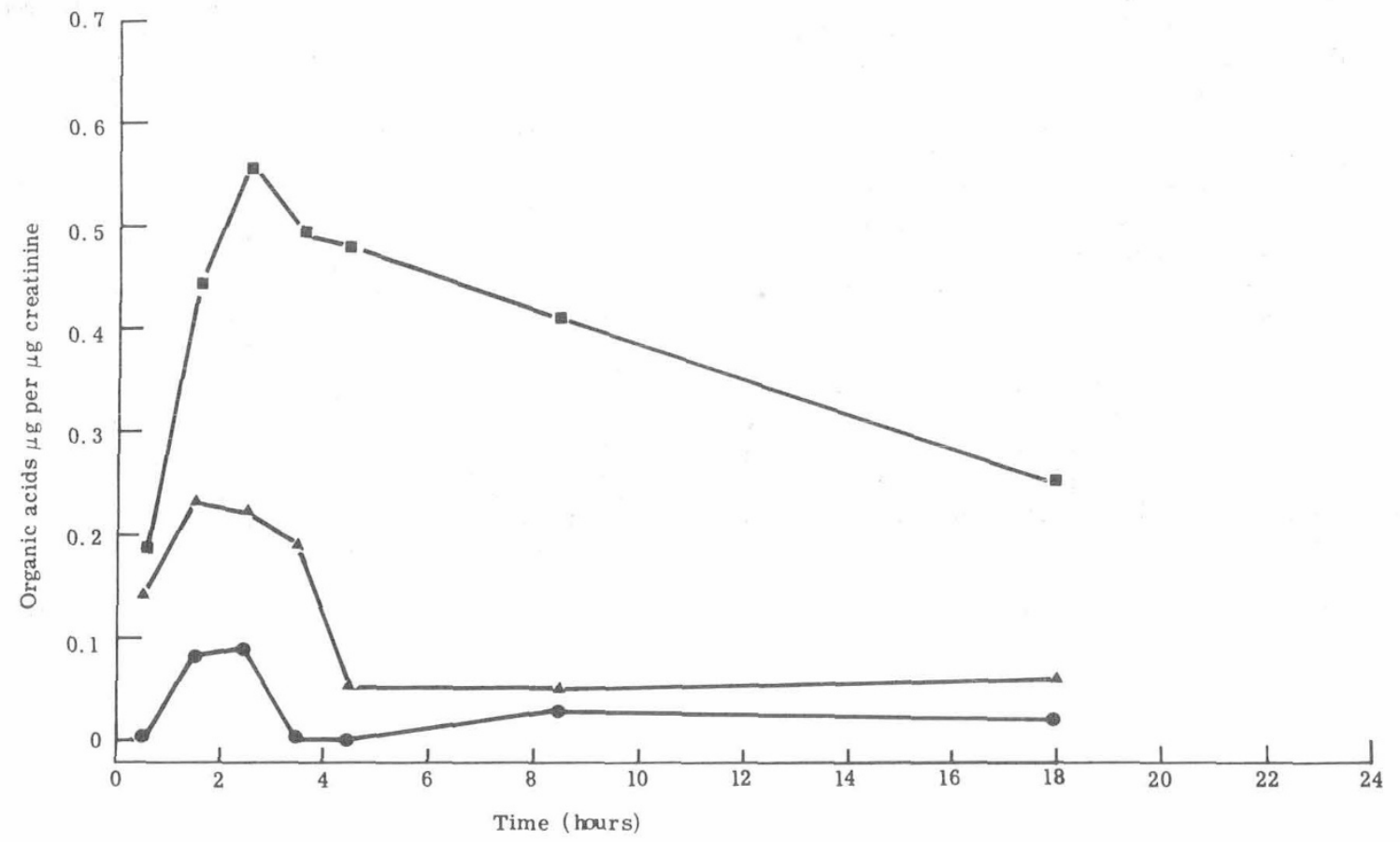

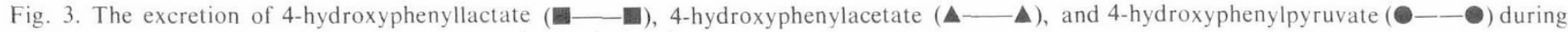
the $24 \mathrm{hr}$ after one oral dose of L-tyrosine ( $100 \mathrm{mg} / \mathrm{kg}$ body weight) on the 199th day of life. The urine collections were made by voluntary voiding and the experimentally determined points are shown at the middle of each urine collection period. 
Table 1. Plasma amino acid concentrations during loading tests with L-phenylalanine and L-tyrosine

\begin{tabular}{|c|c|c|c|c|c|c|}
\hline & \multicolumn{6}{|c|}{ Sample, $\mathrm{mg} / 100 \mathrm{ml}$} \\
\hline & \multicolumn{3}{|c|}{$\begin{array}{l}\text { First phenylalanine load } \\
\text { (202nd day of life) }\end{array}$} & \multicolumn{3}{|c|}{$\begin{array}{c}\text { Tyrosine load } \\
\text { (199th day of life) }\end{array}$} \\
\hline & 0 & $1 \mathrm{hr}$ & $3 \mathrm{hr}$ & $15 \mathrm{~min}$ & $2 \mathrm{hr}$ & $10 \mathrm{hr}$ \\
\hline Phenylalanine & 1.15 & 21.0 & 22.0 & 2.0 & 2.2 & 1.5 \\
\hline Tyrosine & 1.15 & 1.0 & 1.25 & 4.3 & 5.9 & 3.2 \\
\hline
\end{tabular}

to 4-hydroxyphenylpyruvate. This conclusion is consistent with the results of in vitro assays of phenylalanine hydroxylase and tyrosine:2-oxoglutarate amino transferase in patients with hereditary tyrosinemia $(6,13)$.

The recurrence of aromatic aciduria and an abnormal phenylalanine load test when chloral hydrate was reinstituted suggests that the drug or one of its metabolites was responsible for these changes. The other children who were given chloral hydrate did not show an abnormal aromatic acid excretion and we suggest that the patient has an abnormality of the 4-hydroxyphenylpyruvate dioxygenase system which becomes apparent during chloral hydrate administration.

The degree of abnormality in the third phenylalanine load test, which was performed 11 days after stopping chloral hydrate administration (Fig. 2), was less than in the earlier test. The fourth test, which was made after 171 days without chloral hydrate, gave a normal result. These observations indicate progressive improvement in 4-hydroxyphenylpyruvate dioxygenase function which could have been due to withdrawing the drug or to spontaneous recovery of enzyme activity. The abnormal result of the fifth phenylalanine load test (Fig. 2), which was performed near the end of the second period of chloral hydrate administration (Fig. 1), shows clearly that the activity of the enzyme was again reduced. However, the absence of 4-hydroxyphenylpyruvate from all of the urine specimens during the fifth phenylalanine loading test suggests that the degree of enzyme block was less than during the second test, and that the enzyme may have become more resistant to chloral hydrate or chloral hydrate metabolites.

Trichloroacetate is normally a minor metabolite of chloral hydrate, this drug being essentially quantitatively converted to trichloroethanol and trichloroethanolglucuronide $(1,5)$. Trichloroacetate was not found at levels which could be detected by gas chromatography-mass spectrometry in any of the control subjects who were investigated in the present work, and this suggests that the patient may metabolize an abnormally large proportion of a dose of chloral hydrate to this acid.

Inhibition of immature enzyme systems such as 4-hydroxyphenylpyruvate dioxygenase by an unidentified toxin or metabolite could explain the cases in which tyrosinemia has been diagnosed but has slowly reverted to normal (7). These cases do not have the chronic features associated with the type of hereditary tyrosinemia described by Scriver et al. (14).

\section{SUMMARY}

A female infant with the clinical and biochemical features of hereditary tyrosinemia but with spontaneous recovery is described briefly. The results of loading tests with phenylalanine, tyrosine, and sodium 4-hydroxyphenylpyruvate were compatible with a partial block in the oxidation of 4-hydroxyphenylpyruvate to homogentisate which was exacerbated by giving chloral hydrate. The abnormal aromatic carboxylic aciduria was unaltered by giving ascorbic acid. We suggest that an abnormality of the patient's 4-hydroxyphenylpyruvate dioxygenase (EC. 1.13.11.27) system rendered it susceptible to inhibition by chloral hydrate or chloral hydrate metabolites in vivo.

\section{REFERENCES AND NOTES}

1. Breimer, D. D.. Ketelaars, H. C. J., and Van Rossum, J. M.: Gas chromatographic determination of chloral hydrate, trichloroethanol and trichloroacetic acid in blood and urine employing head space analysis. J. Chromatogr., 88: 55 (1974).

2. Chalmers, R. A., Liberman, M., and Watts, R. W. E.: Biochemical studies on at patient with features suggestive of hereditary tyrosinaemia. Clin. Sci. Molec. Med.. 46: 14P (1974).

3. Chalmers, R. A., and Watts, R. W. E.: The quantitative extraction and gas-liquid chromatographic determination of organic acids in urine. Analyst, 97: 958 (1972).

4. Gairdner, D.. and Pearson, J.: A growth chart for premature and other infants, Arch. Dis. Childhood, 46: 783 (1971).

5. Garrett, E. R., and Lambert, H. J.: Pharmacokinetics of trichloroethanol and metabolites and interconversions among various referenced pharmacokinetic parameters. J. Pharmaceut. Sci., 62: 550 (1973)

6. Gaull, G. E., Rassin, D. K., Solomon, G. E., Harris, R. C., and Sturman, J. A.: Biochemical observations on so-called tyrosinemia. Pediat. Res., 4:337 (1970).

7. Harries, J. T., Seakings. J. W. T., Ersser, R. S., and Lloyd, J. K.: Recovery after dietary treatment of an infant with features of tyrosinosis. Arch. Dis. Childhood, 44: 258 (1969).

8. Healy, M J, R Chalmers, R. A and Watts, R. W. E. Reduction of data from the automated gas-liquid chromatographic analysis of complex extracts from human biological fluids using a digital electronic integrator and an off-line computer programme. J. Chromatogr., 87: 365 (1973).

9. Kennaway, N. G., and Buist, N. R. M. Metabolic studies in a patient with hepatic cytosol tyrosine aminotransferase deficiency. Pediat. Res.. 5: 287 (1971).

0. La Du, B, N and Gjessing, L, R. Tyrosinosis and tyrosinaemia, In: J, B. Stanbury, J. B. Wyngaarden, and D. S. Fredrickson: The Metabolic Basis of Inherited Disease, Ed. 3 (McGraw-Hill, New York, 1972).

11. Lawson, A. M., Chalmers, R. A., and Watts, R. W. E.: Studies of O-substituted oxime-trimethylsilyl ester derivatives of some metabolically-important oxocarboxylic acids. Biomed. Mass Spectrom., I: 199 (1974).

12. Medes, G.: A new error of tyrosine metabolism: Tyrosinosis. The intermediary metabolism of tyrosine and phenylalanine. Biochem. J.. 26:917 (1932).

13. Nützenadel, W., Lutz. P.., and Bickel, H.: Primäre und secondäre biochemische veranderungen. Z. Kinderheilk., 113: 193 (1972).

14. Scriver, C. R., Larochelle, J., and Silverberg, M.: Hereditary tyrosinaemia and tyrosyluria in a French Canadian geographic isolate. Amer. J. Dis. Child., 113: 41 (1967).

15. These investigations were approved by the Ethical Committee of Northwick Park Hospital and Clinical Research Centre.

16. We are greatly indebted to the Sisters and nursing staff of Carroll ward and to the pediatric house staff for their unfailing help with the clinical studies. The help of Miss Elaine Bell and Mr. P. Fiveash with the gas chromatography and mass spectrometry, respectively, is also gratefully acknowledged. We are also pleased to acknowledge our indebtedness to Dr. V. G. Oberholzer (The Queen Elizabeth Hospital for Children, Hackney Road. London) for the amino acid analyses.

17. Requests for reprints should be addressed to: R. W. E. Watts, M.D. Head of the Division of Inherited Metabolic Diseases, Clinical Research Centre. Watford Rd., Harrow, Middlesex, HAI 3UJ (England).

18. Accepted for publication July 10, 1975 